\title{
Social complexity: patterns, processes, and evolution
}

\author{
Peter M. Kappeler ${ }^{1,2}$ (D) $\cdot$ Tim Clutton-Brock ${ }^{3} \cdot$ Susanne Shultz ${ }^{4} \cdot$ Dieter Lukas $^{5}$
}

Received: 17 August 2018 / Revised: 11 September 2018 / Accepted: 12 November 2018 / Published online: 19 January 2019

(C) Springer-Verlag GmbH Germany, part of Springer Nature 2019

\begin{abstract}
Animal and human societies exhibit extreme diversity in the size, composition and cohesion of their social units, in the patterning of sex-specific reproductive skew, in the nature of parental care, in the form and frequency of cooperation and in their competitive regime, creating a diversity of socially complex societies. However, there is an ongoing debate about whether social complexity is a real, emergent property of a society or whether it only provides a conceptual framework for studying the diversity and evolution of societies. In this introduction to our topical collection, we identify three areas of current research addressing relevant challenges in the study of social complexity. First, previous studies have suffered from a lack of a common conceptual framework, including shared definitions, and existing measures of social complexity do not acknowledge its multiple components and dimensions. Second, most previous studies have ignored intraspecific variation, and the proximate and ultimate determinants of variation in social complexity, as well as their interactions, remain poorly known. Third, comparative studies of social complexity offer opportunities to explore its biological causes and correlates and but it is frequently difficult to identify the causal relationships involved and the development of general insights has been hampered by conceptual and methodological difficulties. In this paper, we briefly characterize these three challenges and offer guidance to the other contributions to this topical collection on social complexity by placing their key results in the context of these three topics.
\end{abstract}

\section{Significance statement}

In this paper, we address the main current challenges in the study of social complexity and combine our brief overview with a synopsis of the other contributions to this topical collection on various aspects of social complexity.

Communicated by T. Bakker

This article is a contribution to the Topical Collection Social complexity: patterns, processes, and evolution - Guest Editors: Peter Kappeler, Susanne Shultz, Tim Clutton-Brock, and Dieter Lukas

Peter M. Kappeler pkappel@gwdg.de

1 Behavioral Ecology \& Sociobiology Unit, German Primate Center, Leibniz Institute for Primatology, Göttingen, Germany

2 Department of Sociobiology/Anthropology, University of Göttingen, Göttingen, Germany

3 Department of Zoology, University of Cambridge, Cambridge, UK

4 School of Earth and Environmental Sciences, University of Manchester, Manchester, UK

5 Department of Human Behavior, Ecology and Culture, Max Planck Institute for Evolutionary Anthropology, Leipzig, Germany

\section{Introduction}

Animal societies exhibit fascinating diversity in the size, composition, and cohesion of their social units, in the patterning of sex-specific reproductive skew, in the nature of parental care, in the form and frequency of cooperation, and in their competitive regime (Clutton-Brock 2016; Rubenstein and Abbot 2017a). Variation in social systems has often been ranked or ordered along gradients to facilitate comparisons of the relative complexity of different societies (e.g., Sherman et al. 1995; Bourke 1999; Anderson and McShea 2001; Whitehead 2008; Avilés and Harwood 2012; Rubenstein et al. 2016; Rubenstein and Abbot 2017a; Lukas and Clutton-Brock 2018), and to explore relationships between measures of social complexity and interspecific variation in cognition (Barrett et al. 2007; Healy and Rowe 2007; Dunbar and Shultz 2017) or communication (Blumstein and Armitage 1998; Freeberg et al. 2012; Pika 2017). However, it is still an open question whether social complexity is a property of a society that is measureable on a single scale and whether it 
is useful to attempt to compare the complexity of different societies.

A fundamental problem is that social complexity has proved difficult to define and operationalize (Freeberg et al. 2012; Bergman and Beehner 2015; Fischer et al. 2017). In some cases, biologists have regarded more complex societies as those simply consisting of many individuals (Dunbar 1992; Anderson and McShea 2001; Freeberg et al. 2012); others have regarded more complex societies as those where groups are comprised of social roles, such as multiple breeding females, breeders and non-breeding workers or helpers, or members of morphologically different castes (Anderson and McShea 2001; Groenewould et al. 2016; Rubenstein and Abbot 2017b). Yet others regard complex societies as those where social groupings can be identified at multiple levels (Whitehead 2008). Finally, some regard complex societies as those where social relationships between group members are individually differentiated (Bergman and Beehner 2015). Different indices of complexity are not necessarily correlated with each other: indeed, one recent analysis of measures of social complexity across mammals showed that two common measures of social complexity (the extent to which group members occupy functionally different roles and the complexity of interactions between dyads) are negatively correlated with each other (Lukas and Clutton-Brock 2018).

As a result, it is still uncertain whether it is either possible or useful to compare the overall complexity of different societies and there is a need to examine the usage and distribution of specific social traits used to estimate complexity and their relationships to each other. In this paper, we briefly outline some of the current key questions and topics in the study of social complexity, thereby providing a context for the other contributions to this topical collection.

\section{Concepts and methods for studying social complexity}

Characterizing social complexity is fraught with practical and conceptual problems. A main practical problem in this context is due to the fact that both historically and taxonomically, different traits have been used to characterize or rank the degree of social complexity of different taxa (Kappeler 2019, topical collection on social complexity; Lukas and CluttonBrock 2018). In this context, it remains an open question whether it is possible to construct a single index of social complexity that can be applied across taxonomically diverse species or whether different indices of social complexity have to be used in different lineages.

Because comparisons of single traits do not capture all dimensions of social complexity recognized in different studies, multiple aspects of complexity need to be considered whenever possible. It is consequently necessary to decide what range of measures should be included in both single and comparative studies. It might even be feasible to combine several measures into a single estimate of social complexity (see Turchin et al. 2018). Conceptual clarity about relevant measures of social complexity and their inter-dependence is therefore an important prerequisite for comparative research.

Several contributions to this topical collection contribute theoretically or empirically to recent attempts at better describing patterns of social complexity. Kappeler (2019, topical collection on social complexity) critically reviews definitions and previous studies of social complexity in invertebrate and vertebrate societies, and defines social organization, social structure, mating system, and care system as distinct components of any species' social system. He argues that recognition of these four components might offer a framework for comprehensive and comparative studies of social complexity that ought to be useful for future studies.

Aureli and Schino (2019, topical collection on social complexity) add an important perspective by arguing that the study of social complexity can be either based on how it is perceived from the outside or on how it is experienced from within, i.e., how individual group members may experience and perceive the complexity of their social interactions. Focusing on the latter perspective, they propose that variation between and within social relationships, variation in opportunities to interact with different group members, and the variable role of third parties generate species differences in experienced social complexity that may also inform future studies of social cognition.

The contribution by Weiss et al. (2019, topical collection on social complexity) deals with the practical challenges of measuring social complexity. They propose a new method for examining the complexity of animal social networks based on association indices, which can reflect different types of dyadic relationship within a social network. Using binomial mixture models, they examine the performance of this measure with simulated and real data sets, and they outline additional approaches that build on interaction rate and multidimensional relationship data.

Wilkinson et al. (2019, topical collection on social complexity) also use social network analyses, but in combination with genetic data, to determine whether kinship explains the highly variable patterns of association found among bats. Using all available empirical data, they find that most species show evidence of emergent social organization. Specifically, sex-biased dispersal gives rise to interspecific variation in the number of relatives across social units, and bat species in which individuals change roosts frequently tend to exhibit higher levels of association among female relatives. Because the existence of dominance hierarchies and various types of cooperation are independent of the patterns of relatedness across species, the authors conclude that kinship is not a prerequisite for social complexity in bats. 
Finally, Storms et al. (2019, topical collection on social complexity) present empirical data on complex patterns of collective motion exhibited by starlings under attack by a raptor. Collective movements represent one dimension of social organization that can exhibit highly complex dynamics in large animal schools, flocks, or herds. Based on time-series analyses, the authors show that the specific type of collective escape in the three-dimensional environment of their particular study system depends both on the collective pattern that precedes it and the level of threat posed by the raptor.

\section{Variation in social complexity}

Social complexity-however measured - is highly variable across species, which differ in group size, adult sex ratio, and in the nature and patterning of associations and social relationships, for example. Questions concerning the distribution as well as the determinants of this variation are either in the focus of current research or represent important open questions for future research.

First, variation in the components of social systems remains poorly studied. Except for human societies, we know surprisingly little about the magnitude of intraspecific variation in different social traits across populations of the same species inhabiting different habitats, among neighboring groups within a population, or within groups over time (but see Lott 1991; Richards et al. 2003; Jones et al. 2007; Schradin 2013). Moreover, different components of social systems appear to have different phylogenetic inertia (Kamilar and Cooper 2013; Kamilar and Baden 2014), but the nature and magnitude of this variation remain to be systematically explored. For example, whether a species exhibits only maternal or paternal, biparental, cooperative, or no parental care does not seem to vary much within species or even genera and families (Clutton-Brock 1991; Shultz et al. 2011), whereas intraspecific variation in aspects of social organization, like group size and composition, appears to be common and widespread (e.g., Lott 1991; Schradin 2013; Garber et al. 2016; Agnani et al. 2018). Systematic description of the patterning of intra- and interspecific variation among traits characterizing social organization and social structure as well as the mating and care system would seem to provide a basis for both, attempts at better describing social complexity of a given species and for better characterizing mean tendencies of particular traits used in comparative analyses.

Second, much remains to be learned about the factors driving variation in traits contributing to measures of social complexity and their interaction. For example, the study of molecular pathways proximately mediating the control of traits used to assess aspects of social complexity, like alloparental care, from genome structure and gene expression patterns, through epigenetic and developmental processes to mechanisms of neuroendocrine regulation and neural circuits, has so far been largely limited to a few insect model systems (Robinson et al. 2005; Rubenstein and Hofmann 2015; Kapheim 2016; Dogantzis et al. 2018) and should clearly be expanded to suitable vertebrate species.

Recent comparative studies revealed that various ecological and life history variables also impact measures of social complexity. For example, the evolution of cooperative breeding in mammals has been restricted to lineages where females produce multiple offspring per birth, mating systems are monogamous and average coefficients of relatedness between group members are relatively high (Lukas and CluttonBrock 2012, 2018). Similarly, evolutionary transitions from family units to cooperative breeding in birds are associated with higher environmental uncertainty (Jetz and Rubenstein 2011) and shifts to habitats with more variable productivity and more pronounced seasonality (Griesser et al. 2017). In shrimp, ecological generalism is associated with evolutionary transitions to eusociality (Brooks et al. 2017), indicating that sociality is shaped by various ecological factors.

Several contributions to this topical collection address relevant topics in this context. He et al. (2019, topical collection on social complexity) emphasize the role of the physical configuration of habitat features in shaping the social organization and social structure of group-living animals. The logic of their argument involves two steps: by shaping individual decisions about when and where to move, the physical environment impacts which individuals aggregate or encounter one another, and thus their propensity to interact with each other. The authors summarize published studies supporting this neglected perspective and present analytical approaches based on social network measures that can identify and quantify the effects of habitat configuration on social organization and social structure.

Two other contributions to this topical collection highlight the effects of social variables and mechanisms on social complexity, focusing on evolutionary changes and contemporary variation in humans, respectively. van Schaik et al. (2019, topical collection on social complexity) highlight the importance of cumulative culture, which relies fundamentally on social learning, in generating social and cultural complexity in humans. Exploiting various sources of data, the authors argue that the complex forms of cumulative culture characterizing modern humans depended on the simultaneous emergence of language and an increase in proactive cooperation. According to their analyses, the more recent explosive diversification of human social complexity and material culture was triggered by an increase in novelty-seeking, which first facilitated the dramatic range expansions by our ancestors into highly divergent types of habitats and later the emergence of agriculture. This paper therefore nicely emphasizes the temporal dimension of changes in social complexity that is typically not accessible in studies of animal behavior. 
The contribution by Naess et al. (2019, topical collection on social complexity) highlights variation in one aspect of human social complexity (cooperative decision-making) within and across populations. Using gift games to probe cooperative tendencies among pastoralists from independent populations, the authors found that most variance in gift-giving between study sites was due to differences in the importance of relatedness and reciprocity. Their study also illustrates complex interactions among social organization and social structure that may also be found in some animal societies.

Biologically relevant variation in social complexity must ultimately be accompanied by changes in the genetic underpinnings of the relevant traits. Taking an exemplary comprehensive perspective, Kapheim (2019, topical collection on social complexity) reviews recent findings regarding the mechanisms, ontogeny, evolution, and function of social complexity in hymenopterans. Most of our current understanding of the genetic bases of traits impacting social systems is based on studies of members of this lineage, and this contribution provides a welcome accessible introduction to this topic. It is also becoming increasingly apparent that developmental plasticity, e.g., maternal or sibling effects on development, are major drivers of caste-related behavior, i.e., social cues also act as proximate determinants of social variation. How social and molecular traits interact to generate variation and ultimately evolutionary change in social complexity in vertebrates is a frontier in the study of sociality (Rubenstein and Hofmann 2015) that will be informed and inspired by the existing body of research summarized in Kapheim's paper.

\section{Evolutionary changes in social complexity}

It is equally interesting to explore the evolutionary correlates and consequences of changes in measures of social complexity and other traits, such as cognitive abilities, brain size, or communication signals. We have already emphasized the importance of choosing clearly defined variables representing variation in social complexity for both, assembling data sets with high internal validity for comparative studies based on multiple published sources and for meaningful comparison across studies of different taxa or independent studies of the same lineage (see also Borries et al. 2016; Lukas and CluttonBrock 2017; Schradin 2017). A second prerequisite concerns the quality of the phylogenetic information that is used to either reconstruct ancestral states and trait evolution or to control for phylogenetic non-independence as well as choosing appropriate comparative methods (Garamszegi 2014).

In terms of the biologically important questions in this context, there has been discussion of (i) which traits constitute components of social complexity and which ones are independent of each other, (ii) whether components of social complexity vary categorically or continuously across species, (iii) the direction(s) of causality in any co-evolutionary pattern detected through comparative analyses, and (iv) the relative importance of any singular measure of social complexity in explaining variation in a given other trait of interest.

The remaining contributions to this topical collection address at least one of these issues in this very active field of research. Peckre et al. (2019, topical collection on social complexity) revisit the relationship between social and communicative complexity. Several, but not all, previous studies had suggested that animals living in more complex social environments also have communication repertoires with more and/or more complex signals. The authors first establish problems with the operationalization of measures of both variables, including the neglect of the multi-modal nature of most social communication, that may have contributed to equivocal outcomes across studies. They go on to emphasize the importance of also considering alternative hypotheses to social complexity in explaining covariation in other traits; in this case, the relative roles of ecology, morphology and phylogenetic history in driving signal complexity. As indicated by their discussion of the intimate relationships between social context, including the presence of variable audiences, and signaling behavior, one may even question the conceptual separation between social and communicative complexity (as also argued by Kappeler 2019, topical collection on social complexity).

By detailed comparisons at two levels, i.e., between different social contexts of signal production and between closely related species, Gustison et al. (2019, topical collection on social complexity) provide an example of how differences in social organization and social structure between two species of Old World monkeys are accompanied by changes in their respective vocal repertoires. Geladas, which live in very large groups, produce vocalizations in long sequences, especially when levels of conspecific noise are high. Sequence complexity, but not duration, increase when males approached females to initiate social interactions. Thus, compared to chacma baboons, the change in social organization (group size) and social structure (cross-sex bonds) towards more complexity has led to corresponding changes in vocal complexity. The exact co-evolutionary dynamics, i.e., "have changes in X permitted, facilitated, driven or followed changes in Y?", remain also obscure at this very detailed level of comparison, however.

The contribution by Boucherie et al. (2019, topical collection on social complexity) emphasizes the value of in-depth study of other taxa exhibiting interesting variation in social complexity and cognitive abilities apart from primates. Reviewing decades of research on natural social dynamics and experimentally probed socialcognitive skills of ravens, the authors show how these traits co-vary. Their focus on a comprehensive set of 
studies conducted on this single species also leads them to emphasize the importance of how being intimately familiar with a particular species can improve the detail and biological relevance of questions about the evolution of social complexity being asked.

The contribution by Fuentes-González and Martins (2019, topical collection on social complexity) focuses on the analytical methods used in studies of the evolution of social complexity and its co-evolution with other traits. They briefly review some of the advances and pitfalls in the statistical approaches underlying such analyses. They then illustrate the usefulness of a new method by analyzing two aspects of social complexity in primates: variation in group size as a function of activity pattern and habitat use and variation in mating systems driving the evolution of baculum length. Thus, this paper also illustrates that aspects of social complexity co-evolve with many other traits and that they can be treated as both independent or dependent variables.

Taken together, the contributions to this topical collection on social complexity sketch a representative picture of the diversity of questions and approaches in current research on this topic. We hope that this topical collection will stimulate additional research on key problems in the study of animal and human societies within the framework of social complexity.

Acknowledgments We thank the German Research Foundation (DFG) for supporting the conference on "Social complexity: Patterns, Processes and Evolution," which took place in Göttingen in December 2017, and we are grateful to the referees of the contributions to this topical collection for sharing their insights and expertise.

\section{Compliance with ethical standards}

Conflict of interest The authors declare that they have no conflict of interest.

Publisher's Note Springer Nature remains neutral with regard to jurisdictional claims in published maps and institutional affiliations.

\section{References}

Agnani P, Kauffmann C, Hayes Loren D, Schradin C (2018) Intraspecific variation in social organization of Strepsirrhines. Am J Primatol 80:e22758

Anderson C, McShea DW (2001) Individual versus social complexity, with particular reference to ant colonies. Biol Rev 76:211-237

Aureli F, Schino G (2019) Social complexity from within: how individuals experience the structure and organization of their groups. Behav Ecol Sociobiol. https://doi.org/10.1007/s00265-018-2604-5

Avilés L, Harwood G (2012) A quantitative index of sociality and its application to group-living spiders and other social organisms. Ethology 118:1219-1229

Barrett L, Henzi P, Rendall D (2007) Social brains, simple minds: does social complexity really require cognitive complexity? Phil Trans $\mathrm{R}$ Soc B 362:561-575
Bergman TJ, Beehner JC (2015) Measuring social complexity. Anim Behav 103:203-209

Blumstein DT, Armitage KB (1998) Life history consequences of social complexity: a comparative study of ground-dwelling sciurids. Behav Ecol 9:8-19

Borries C, Sandel AA, Koenig A, Fernandez-Duque E, Kamilar JM, Amoroso CR, Barton RA, Bray J, di Fiore A, Gilby IC, Gordon AD, Mundry R, Port M, Powell LE, Pusey AE, Spriggs A, Nunn CL (2016) Transparency, usability, and reproducibility: guiding principles for improving comparative databases using primates as examples. Evol Anthropol 25:232-238

Boucherie PH, Loretto MC, Massen JJM, Bugnyar T (2019) What constitutes 'social complexity' and 'social intelligence' in birds? Lessons from ravens. Behav Ecol Sociobiol. https://doi.org/10. 1007/s00265-018-2607-2

Bourke AFG (1999) Colony size, social complexity and reproductive conflict in social insects. J Evol Biol 12:245-257

Brooks KC, Maia R, Duffy JE, Hultgren KM, Rubenstein DR (2017) Ecological generalism facilitates the evolution of sociality in snapping shrimps. Ecol Lett 20:1516-1525

Clutton-Brock TH (1991) The evolution of parental care. Princeton University Press, Princeton

Clutton-Brock TH (2016) Mammal societies. John Wiley \& Sons, Chichester

Dogantzis KA, Harpur BA, Rodrigues A, Beani L, Toth AL, Zayed A (2018) Insects with similar social complexity show convergent patterns of adaptive molecular evolution. Sci Rep 8:10388

Dunbar RIM (1992) Neocortex size as a constraint on group size in primates. J Hum Evol 20:469-493

Dunbar RIM, Shultz S (2017) Why are there so many explanations for primate brain evolution? Phil Trans R Soc B 372:20160244

Fischer J, Farnworth MS, Sennhenn-Reulen H, Hammerschmidt K (2017) Quantifying social complexity. Anim Behav 130:57-66

Freeberg TM, Dunbar RIM, Ord TJ (2012) Social complexity as a proximate and ultimate factor in communicative complexity. Phil Trans R Soc B 367:1785-1801

Fuentes-González JA, Martins EP (2019) Phylogenies and social complexity. Behav Ecol Sociobiol. https://doi.org/10.1007/s00265-0182614-3

Garamszegi LZ (ed) (2014) Modern phylogenetic comparative methods and their application in evolutionary biology. Springer, Heidelberg

Garber PA, Porter LM, Spross J, Di Fiore A (2016) Tamarins: insights into monogamous and non-monogamous single female social and breeding systems. Am J Primatol 78:298-314

Griesser M, Drobniak SM, Nakagawa S, Botero CA (2017) Family living sets the stage for cooperative breeding and ecological resilience in birds. PLoS Biol 15:e2000483

Groenewoud F, Frommen JG, Josi D, Tanaka H, Jungwirth A, Taborsky M (2016) Predation risk drives social complexity in cooperative breeders. P Natl Acad Sci USA 113:4104-4109

Gustison M, Johnson ET, Beehner JC, Bergman TJ (2019) The social functions of complex vocal sequences in wild geladas. Behav Ecol Sociobiol. https://doi.org/10.1007/s00265-018-2612-5

He P, Malonado-Chaparro A, Farine DR (2019) The role of habitat configuration in shaping social structure: a gap in studies of animal social complexity. Behav Ecol Sociobiol. https://doi.org/10.1007/ s00265-018-2602-7

Healy SD, Rowe C (2007) A critique of comparative studies of brain size. Proc R Soc Lond B 274:453-464

Jetz W, Rubenstein DR (2011) Environmental uncertainty and the global biogeography of cooperative breeding in birds. Curr Biol 21:72-78

Jones TC, Riechert SE, Dalrymple SE, Parker PG (2007) Fostering model explains variation in levels of sociality in a spider system. Anim Behav 73:195-204

Kamilar JM, Baden AL (2014) What drives flexibility in primate social organization? Behav Ecol Sociobiol 68:1677-1692 
Kamilar JM, Cooper N (2013) Phylogenetic signal in primate behaviour, ecology and life history. Phil Trans R Soc B 368:20120341

Kapheim KM (2016) Genomic sources of phenotypic novelty in the evolution of eusociality in insects. Curr Opin Insect Sci 13:24-32

Kapheim KM (2019) Synthesis of Tinbergen's four questions and the future of sociogenomics. Behav Ecol Sociobiol. https://doi.org/10. 1007/s00265-018-2606-3

Kappeler PM (2019) A framework for studying social complexity. Behav Ecol Sociobiol. https://doi.org/10.1007/s00265-018-2601-8

Lott DF (1991) Intraspecific variation in the social systems of wild vertebrates. Cambridge University Press, Cambridge

Lukas D, Clutton-Brock TH (2012) Life histories and the evolution of cooperative breeding in mammals. Proc R Soc Lond B 279:40654070

Lukas D, Clutton-Brock T (2017) Comparative studies need to rely both on sound natural history data and on excellent statistical analysis. $\mathrm{R}$ Soc Open Sci 4:171211

Lukas D, Clutton-Brock TH (2018) Social complexity and kinship in animal societies. Ecol Lett 21:1129-1134

Naess MW, Du J, Thomas MG, Bardsen BJ, Mace R (2019) Comparison of social complexity in two independent pastoralist societies. Behav Ecol Sociobiol. https://doi.org/10.1007/s00265-018-2611-6

Peckre L, Kappeler PM, Fichtel C (2019) Clarifying and expanding the social complexity hypothesis for communicative complexity. Behav Ecol Sociobiol. https://doi.org/10.1007/s00265-018-2605-4

Pika S (2017) Unpeeling the layers of communicative complexity. Anim Behav 134:223-227

Richards MH, von Wettberg EJ, Rutgers AC (2003) A novel social polymorphism in a primitively eusocial bee. P Natl Acad Sci USA 100: $7175-7180$

Robinson GE, Grozinger CM, Whitfield CW (2005) Sociogenomics: social life in molecular terms. Nat Rev Genet 6:257-270

Rubenstein DR, Abbot P (2017a) Comparative social evolution. Cambridge University Press, Cambridge, UK

Rubenstein DR, Abbot P (2017b) Social synthesis: opportunities for comparative social evolution. In: Rubenstein DR, Abbot P (eds) Comparative social evolution. Cambridge University Press, Cambridge, UK, pp 427-452

Rubenstein DR, Hofmann HA (2015) Proximate pathways underlying social behavior. Curr Opin Behav Sci 6:154-159
Rubenstein DR, Botero CA, Lacey EA (2016) Discrete but variable structure of animal societies leads to the false perception of a social continuum. R Soc open sci 3:160147

Schradin C (2013) Intraspecific variation in social organization by genetic variation, developmental plasticity, social flexibility or entirely extrinsic factors. Phil Trans R Soc B 368:20120346

Schradin C (2017) Comparative studies need to rely both on sound natural history data and on excellent statistical analysis. R Soc open sci 4:170346

Sherman PW, Lacey EA, Reeve HK, Keller L (1995) The eusociality continuum. Behav Ecol 6:102-108

Shultz S, Opie C, Atkinson QD (2011) Stepwise evolution of stable sociality in primates. Nature 479:219-222

Storms R, Carere C, Zoratto F, Hemelrijk CK (2019) Complex collective motion: escape patterns in starling flocks under predation. Behav Ecol Sociobiol. https://doi.org/10.1007/s00265-018-2609-0

Turchin P, Currie TE, Whitehouse H, François P, Feeney K, Mullins D, Hoyer D, Collins C, Grohmann S, Savage P, Mendel-Gleason G, Turner E, Dupeyron A, Cioni E, Reddish J, Levine J, Jordan G, Brandl E, Williams A, Cesaretti R, Krueger M, Ceccarelli A, Figliulo-Rosswurm J, Tuan PJ, Peregrine P, Marciniak A, PreiserKapeller J, Kradin N, Korotayev A, Palmisano A, Baker D, Bidmead J, Bol P, Christian D, Cook C, Covey A, Feinman G, Júlíusson ÁD, Kristinsson A, Miksic J, Mostern R, Petrie C, Rudiak-Gould P, ter Haar B, Wallace V, Mair V, Xie L, Baines J, Bridges E, Manning J, Lockhart B, Bogaard A, Spencer C (2018) Quantitative historical analysis uncovers a single dimension of complexity that structures global variation in human social organization. P Natl Acad Sci USA 115:e144-e151

van Schaik CP, Pradhan GR, Tennie C (2019) The evolution of cultural complexity in the hominin lineage. Behav Ecol Sociobiol. https:// doi.org/10.1007/s00265-018-2610-7

Weiss M, Franks DW, Croft DP, Whitehead H (2019) Measuring the complexity of social associations using mixture models. Behav Ecol Sociobiol. https://doi.org/10.1007/s00265-018-2603-6

Whitehead H (2008) Analyzing animal societies. Chicago University Press, Chicago

Wilkinson GS, Carter GG, Bohn KM et al (2019) Kinship, association and social complexity in bats. Behav Ecol Sociobiol. https://doi.org/ 10.1007/s00265-018-2608-1 